\title{
A Novel Hypogenetic Chaotic Jerk System: Modeling, Circuit Implementation, and Its Application
}

\author{
Jiancheng Liu, ${ }^{1}$ Karthikeyan Rajagopal $\mathbb{D},{ }^{2}$ Tengfei Lei $\mathbb{D}^{3},{ }^{3}$ Sezgin Kaçar, ${ }^{4}$ BurakArıcıoğlu, ${ }^{4}$ \\ Ünal Çavuşoğlu, ${ }^{5}$ Abdullah Hulusi Kökçam $\left(\mathbb{1},{ }^{6}\right.$ and Anitha Karthikeyan ${ }^{7}$ \\ ${ }^{1}$ Jiangsu Key Laboratory of Meteorological Observation and Information Processing, \\ Nanjing University of Information Science \& Technology, Nanjing 210044, China \\ ${ }^{2}$ Nonlinear Systems and Applications, Faculty of Electrical and Electronics Engineering, Ton Duc Thang University, \\ Ho Chi Minh City, Vietnam \\ ${ }^{3}$ School of Mechanical and Electrical Engineering, Qilu Institute of Technology, Jinan 250200, China \\ ${ }^{4}$ Department of Electrical and Electronics Engineering, Faculty of Technology, Sakarya University, Serdivan, Sakarya, Turkey \\ ${ }^{5}$ Department of Computer Engineering, Faculty of Computer and Information Sciences, Sakarya University, Serdivan, \\ Sakarya, Turkey \\ ${ }^{6}$ Department of Industrial Engineering, Faculty of Engineering, Sakarya University, Serdivan, Sakarya, Turkey \\ ${ }^{7}$ Nonlinear Systems and Applications, Faculty of Electrical and Electronics Engineering, Ton Duc Thang University, \\ Ho Chi Minh City, Vietnam
}

Correspondence should be addressed to Tengfei Lei; leitengfeicanhe@126.com

Received 23 February 2020; Revised 28 March 2020; Accepted 6 April 2020; Published 4 May 2020

Guest Editor: Ping Zhao

Copyright ( 2020 Jiancheng Liu et al. This is an open access article distributed under the Creative Commons Attribution License, which permits unrestricted use, distribution, and reproduction in any medium, provided the original work is properly cited.

When revising the polarity and amplitude information in the feedback, a unique hypogenetic jerk system was obtained which has two controllers to switch the equilibria between stable and unstable. After providing some basic dynamical analysis, an electronic circuit was implemented, and the phase trajectory in the oscilloscope agrees with the numerical simulation. Further exploration shows that this unique chaotic system has superior performance as a random number generator or in voice encryption application.

\section{Introduction}

In the literature, chaos has a wide range of application field. In the recent years, chaos or chaotic systems have been frequently employed in the encryption and random number generation (RNG) studies due to noise-like, aperiodic characteristics of the chaotic systems [1]. In the literature, the encryption studies are not only about image encryption [2-5] but also there are encryption studies about text and other multimedia types like audio and video encryption [6]. It shows that chaos has great performance in the encryption system. In fact, RNG studies in the literature can be categorized into true random number generation (TRNG) and pseudo-random number generation (PRNG) regardless of whether they are chaos-based or not $[7,8]$. RNG is usually employed in cryptographic studies for key generation process and performance of the encryption which heavily depends on key randomness. This can be achieved with appropriate chaos-based random number generation process. All these constitute our motivation to develop a new chaotic system.

Jerk dynamical systems are realized with a compact electrical circuit structure. Generally, jerk circuits have four connections at the node $x$, where the derivative of $x$ is determined by the amplitude and polarity of $y$, and the amplitude and polarity of $x$ influence the derivative of $z$. We study those chaotic flows with incomplete information transmission from the node $x$ based on the jerk structure and are therefore named hypogenetic chaotic. Jerk system has the simple structure but can also provide applicable chaotic signal [9-13]. When the feedback information from the other variables is not complete, many of the systems can remain chaos [14-16] even in the jerk structure as 
hypogenetic flows [14]. Hot research on chaotic systems mainly focuses on chaos control [17-20], Lyapunov exponent calculation and analysis $[21,22]$, and doubling and growth of attractors [23, 24], and chaotic systems with hidden attractors are also hot research topics because such systems are extremely prone to multistable phenomena, which are a common phenomenon in nature. Considering from equilibrium point, hidden attractors can be mainly of several types, one stable equilibrium[25], a line or plane equilibrium [26, 27], or no equilibrium [28]. Even there are hidden attractors found in some special chaotic systems [29], which have both unstable equilibrium and stable equilibria.

Starting from the classic system, hidden attractor is a very important topic. At the same time, linearization has always been a fundamental state. Is there a class of system whose linear linearization satisfies the characteristics of the chaotic hidden attractor (stable equilibrium system)? The circuit of this kind of system is less disturbed because linearization (sign circuit) is stable and similar to the digital circuit.

Therefore, we propose a class of hypogenetic chaotic system which has the following unique properties: nonlinearity involved includes amplitude information and polarity information, which are of incomplete feedback; there are two knobs which control the stability of equilibria, by which one can turn off the stable states freely.

This paper is organized as follows. In Section 2, we give a model description including basic dynamical analysis, and in Section 3, we show the electronic circuit implementation of the system. Further application discussions including random number generator design and voice encryption application are given in Section 4. In the last section, we give a simple conclusion.

\section{Model Description}

In this letter, we announce a new jerk system derived by modifying the jerk system proposed in [14] by replacing the nonlinear terms $z^{2}$ by $|z|$ and $x z$ by $z \operatorname{sgn}(x)$. The proposed system shows a unique property of disorder which has both stable and unstable equilibrium points for different values of parameters.

$$
\begin{aligned}
& \dot{x}=a_{1} y, \\
& \dot{y}=a_{2} z, \\
& \dot{z}=a_{3} x+a_{4}|z|+a_{5} x y+a_{6} z \operatorname{sgn}(x)+a_{7},
\end{aligned}
$$

where $a_{i}$ for $i \in[1,7]$ are the parameters of the system. Jerk systems have the form $\left\{\begin{array}{l}\dot{x}=y \\ \dot{y}=z \\ \dot{z}=f(x, y, z)\end{array}\right.$, which can be depicted as $\ddot{x}=f(x, \dot{x}, \ddot{x})$ [30]. When $a_{1}=1$ and $a_{2}=1$, system (1) is Jerk system.

It is simple to verify that the equilibrium point of system (1) is $x=-\left(a_{7} / a_{3}\right), \quad y=0$, and $z=0$, and the characteristic equation of the system is $\lambda^{3}+a_{6} \operatorname{sgn}\left(a_{7} / a_{3}\right) \lambda^{2}+$ $\left(a_{2} a_{5} a_{7} / a_{3}\right) \lambda-a_{1} a_{2} a_{3}=0$. According to the Routh-Hurwitz criterion for the real part of the eigenvalues to be positive, the condition is $\delta_{1}, \delta_{0}>0, \delta_{1} \delta_{2}>\delta_{0}$, where $\delta_{0}=1, \delta_{1}=a_{6} \operatorname{sgn}\left(a_{7} / a_{3}\right), \delta_{2}=\left(a_{2} a_{5} a_{7} / a_{3}\right)$, and $\delta_{3}=-a_{1} a_{2} a_{3}$. For the values of parameters $a_{1}=1, a_{2}=1$, $a_{3}=-1, a_{4}=-4$, and $a_{6}=1$ and to discuss the effect of parameters on the type of equilibrium points, we vary the parameters $a_{5}, a_{7}$ and investigate the type of equilibrium point with the real part of eigenvalues and the Routh-Hurwitz condition $\delta_{1} \delta_{2}>\delta_{0}$. Figure 1 (a) shows the change in the real part of eigenvalues of system (1) with parameter $a_{5}$, and for $a_{5}<1$, the real part of eigenvalues become negative making the equilibrium stable. Similarly, Figure 1(b) is change in the real part of eigenvalues with $a_{7}$, and for $a_{7}>-0.6$, the equilibrium is stable.

To analyze the stability of the systems using Routh-Hurwitz (RH) criterion, we investigate the two conditions $\delta_{1}, \delta_{0}>0$ and $\delta_{1} \delta_{2}>\delta_{0}$. For the parameter values $a_{1}=1, a_{2}=1, a_{3}=-1, a_{4}=-4$, and $a_{6}=1$, the RH condition modifies to $\delta_{0}=1, \delta_{1}=-a_{5} a_{7}$, and $\delta_{2}=-\operatorname{sign}\left(a_{7}\right)$. We vary the parameters $a_{5}$ and $a_{7}$ between $[0.7,2]$ and $[-1.3,-0.6]$, respectively, and the condition $\delta_{1} \delta_{2}>\delta_{0}$ is plotted as shown in Figure 2 which confirms our claim that the equilibrium is stable when $a_{7}>-0.6$ or $a_{5}<1$. Both the eigenvalues and RH investigations confirm that system (1) exhibits both stable (nonhyperbolic) and unstable (hyperbolic) equilibrium points. To the best of our knowledge, this feature has not been investigated in the literature.

To show the 2D phase portraits of the stable and unstable system, we fix $a_{7}=-1$ and plot for $a_{5}=0.95$ (stable equilibrium) and $a_{5}=1.6$ (unstable equilibrium) as shown in Figures 3(a) and 3(b), respectively.

The finite-time Lyapunov exponents of system (1) are derived using Wolf's algorithm [1] for both stable and unstable cases and are given in Table 1 with the Kaplan-Yorke dimension $\left(D_{\mathrm{KY}}\right)$ calculated for a run time of 20,000 s with initial conditions $\left[\begin{array}{lll}-3 & 4 & -4\end{array}\right]$ with other parameters except $a_{5}, a_{7}$ fixed to their respective chaotic values.

To further understand the complete dynamical behavior of the system, we derived the bifurcation plots with parameter $a_{5}$ as it governs the equilibrium points of the proposed system. The parameter $a_{5}$ is varied between $[0.8,2]$, and the local maximum of the state variable $z$ is plotted as shown in Figures 4(a) and 4(b) which show the corresponding LEs of the system. It can be seen from Figure 4(a) that, under the change of parameter $a_{5}$, the chaos state and periodic state of the system appear alternately, and the way the system changes from the periodic state to chaos is period-doubling bifurcation. By comparing Figures 4(a) and $4(\mathrm{~b})$, the maximum Lyapunov exponent of the system is zero; When the system is chaotic, the largest Lyapunov exponent of the system is positive. When the system is convergent, the largest Lyapunov exponent of the system is negative.

\section{Electronic Circuit Implementation of the System}

In this section, system (1) is implemented with an electronic circuit. The circuit designed in PSpice medium and its simulation are carried out. The schematic of the designed 


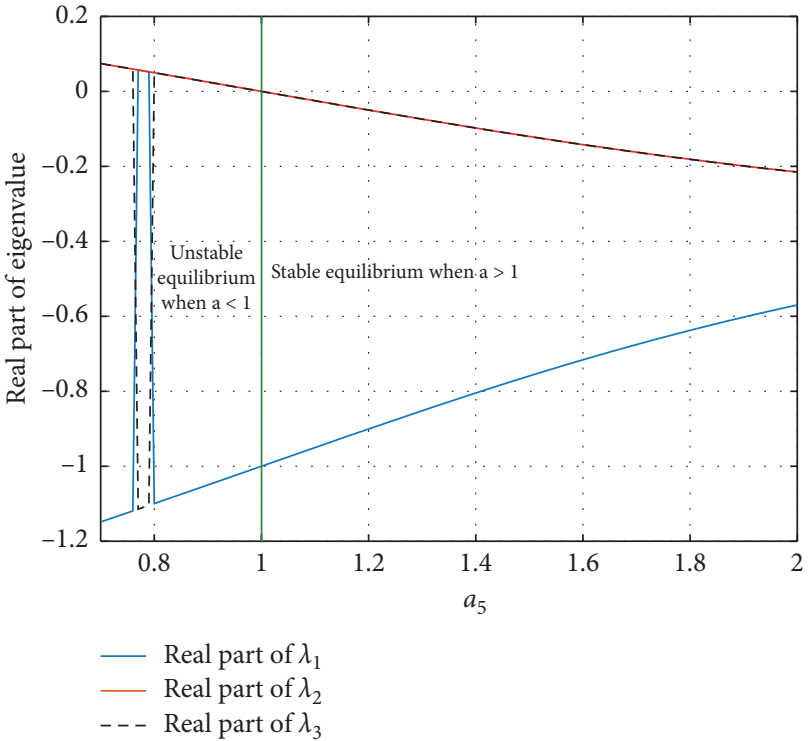

(a)

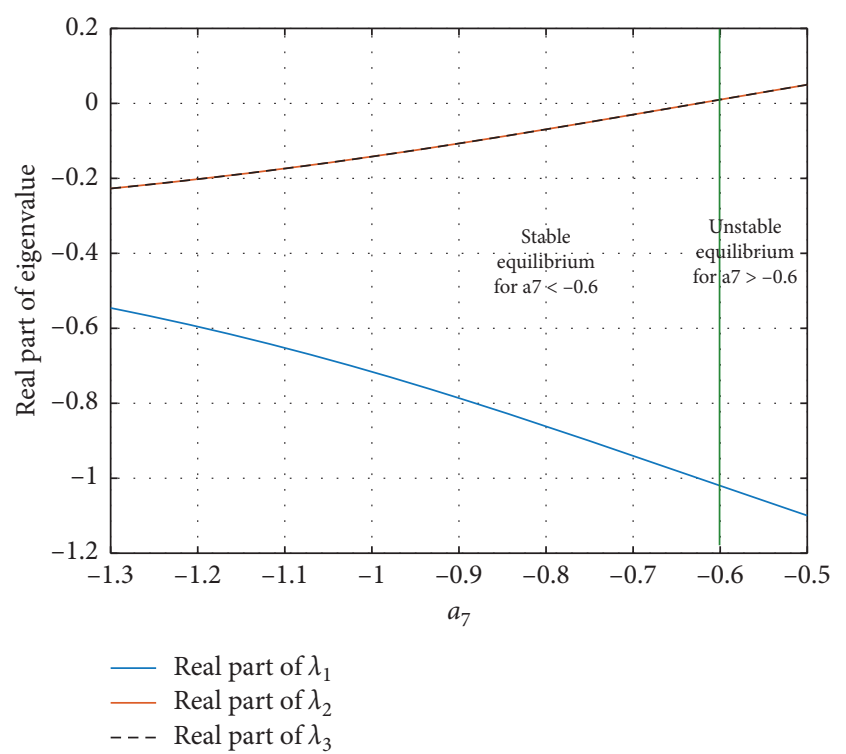

(b)

FIgURe 1: Eigenvalues of the system for various values of (a) $a_{5}$ and (b) $a_{7}$.
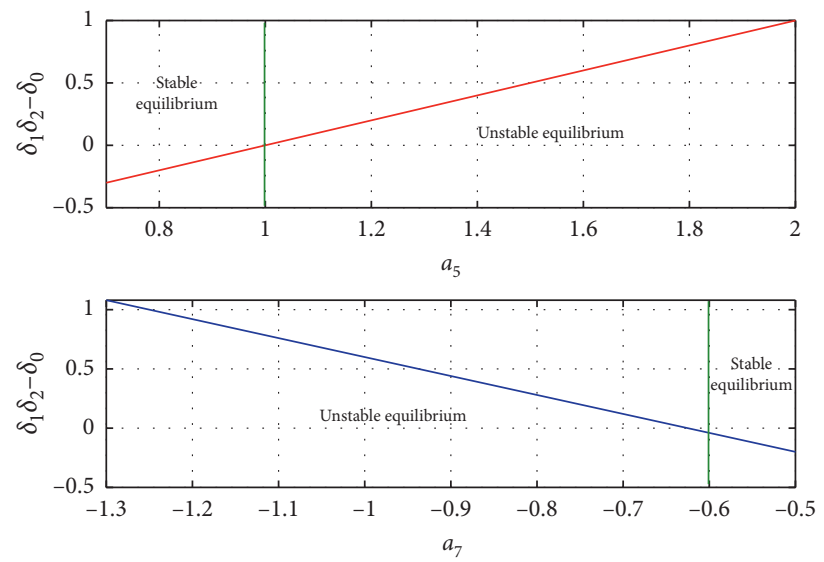

Figure 2: Plots showing the RH condition $\delta_{1} \delta_{2}>\delta_{0}$ vs. parameters $a_{5}$ and $a_{7}$.

circuit is given in Figure 5. Here, we applied the traditional method by the multiplier AD633/AD to carry out the function $z \operatorname{sgn}(\mathrm{x})$, and the new switch published in [25] was also tried by simulation which shows the same results.

The electronic circuit consists of OpAmps, multiplier ICs, diodes, resistors, and capacitors. The supply voltages of the active elements are $+15 \mathrm{~V}$ for positive supply inputs and $-15 \mathrm{~V}$ for negative supply inputs. The value of the resistors used in the circuit is as follows: $R_{1}=R_{2}=R_{3}=400 \mathrm{k} \Omega, R_{4}=$ $R_{8}=R_{9}=R_{12}=R_{13}=R_{14}=R_{15}=R_{16}=100 \mathrm{k} \Omega, \quad R_{5}=42 \mathrm{k} \Omega$, $R_{6}=40 \mathrm{k} \Omega$, and $R_{7}=6 \mathrm{M} \Omega$. The values of the capacitor in the circuit are as follows: $C_{1}=C_{2}=C_{3}=1 \mathrm{nF}$. The given resistor and capacitor values are for the stable equilibrium case when $a_{5}=0.95$ and $a_{7}=-1$. For the unstable equilibrium case (when $a_{5}=1.6$ and $a_{7}=-1$ ), only the value of $R_{5}$ resistor will be changed. The value of $R_{5}$ resistor is $25 \mathrm{k} \Omega$.
The real-time electronic circuit application is also realized. The results of the real-time electronic circuit are examined on the oscilloscope. 2D phase portraits of system (1) obtained from the oscilloscope are given in Figures 6(a) and $6(\mathrm{~b})$. The phase portrait of the stable equilibrium case $\left(a_{5}=\right.$ 0.95 and $a_{7}=-1$ ) is given in Figure 6(a) while that of the unstable equilibrium case $\left(a_{5}=1.6\right.$ and $\left.a_{7}=-1\right)$ is given in Figure $6(\mathrm{~b})$. Both of them confirm the theoretical prediction in Figure (3).

\section{Application Discussion}

4.1. Random Number Generator Design and NIST Tests. Random number generator (RNG) is one of the most important applications in which chaotic systems are used. RNGs are used in many different engineering fields, especially communication and cryptography. In this section, 

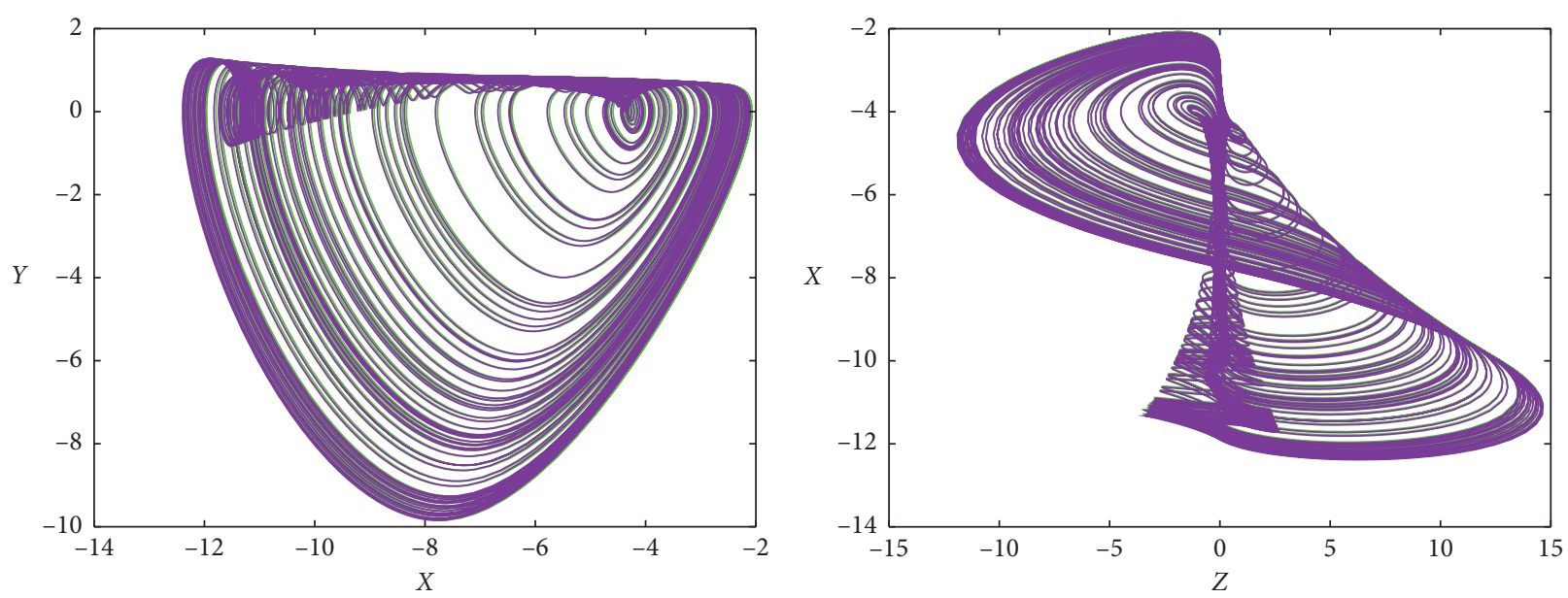

(a)
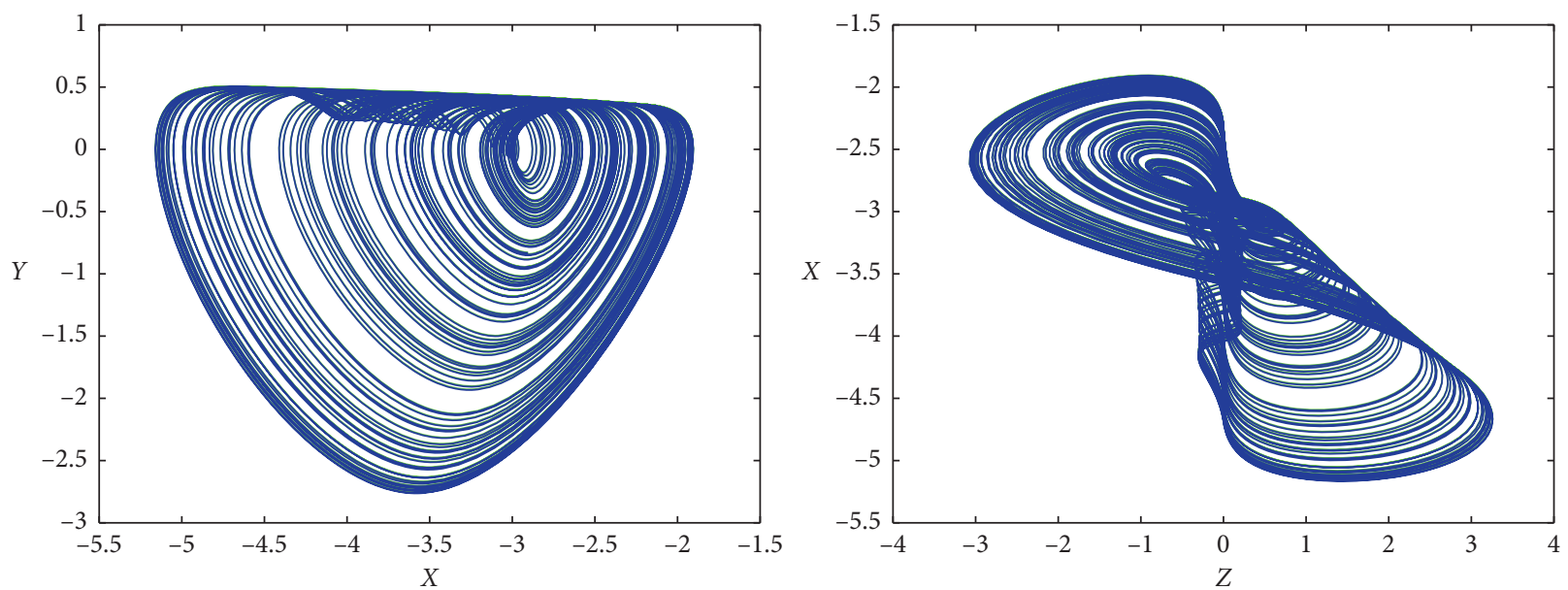

(b)

FIGURE 3: 2D phase portraits of the novel jerk system for $a_{1}=1, a_{2}=1, a_{3}=-1, a_{4}=-4$, and $a_{6}=1$ and initial conditions [ $-34-4$ ]. Figure 3(a) shows system (1) with a stable equilibrium for $a_{5}=0.95$ and $a_{7}=-1$, and Figure 3 (b) shows system (1) with a unstable equilibrium for $a_{5}=1.6$ and $a_{7}=-1$.

TABLE 1: Lyapunov exponents and $D_{\mathrm{KY}}$ for different parameters.

\begin{tabular}{lcc}
\hline Parameters & Lyapunov exponents (LEs) & $D_{\mathrm{KY}}$ \\
\hline$a_{5}=0.95, a_{7}=-1$ & {$[0.1196,0,-1.179]$} & 2.101 \\
$a_{5}=1.6, a_{7}=-1$ & {$[0.0727,0,-1.375]$} & 2.052 \\
\hline
\end{tabular}

a new RNG design has been realized by using the new chaotic jerk system proposed in this study. The flow diagram of the method used in the design of RNG is given in Figure 7.

As shown in the flow diagram, the appropriate initial values, the number of bits taken from the state variables, and the step value for the RK4 algorithm are determined with the help of the new chaotic system. Then, the RK4 algorithm is executed to obtain discrete arrays of state variables and convert them into 32 bit binary arrays. Steps to generate the pseudo-random sequences are as follows.

The system uses the RK4 algorithm and Matlab software to iterate 2,000 times for simulation.
S1: the sequence obtained by the first iteration of the system data $=[x(1), y(1), z(1)]$; the numerical value is kept to the second decimal place.

S2: data $=$ data $* 100$; data are expressed as a 32 bit binary number: data $=\left\{b_{31} b_{30}, \ldots, b_{2} b_{1}\right\}_{32}$.

S3: Let data $1=\left\{b_{7} b_{6}, \ldots, b_{2} b_{1}\right\}_{8}$, data1 for further test use.

S4: $n=n+1$. Then, it is still stated that the sequence to be generated passes through S2-S4 until $n=2001$.

Random bit arrays are generated for NIST tests by taking the appropriate number of bits from these arrays. If the generated bit array passes these tests successfully, the RNG design is completed and ready for use in practical applications. 


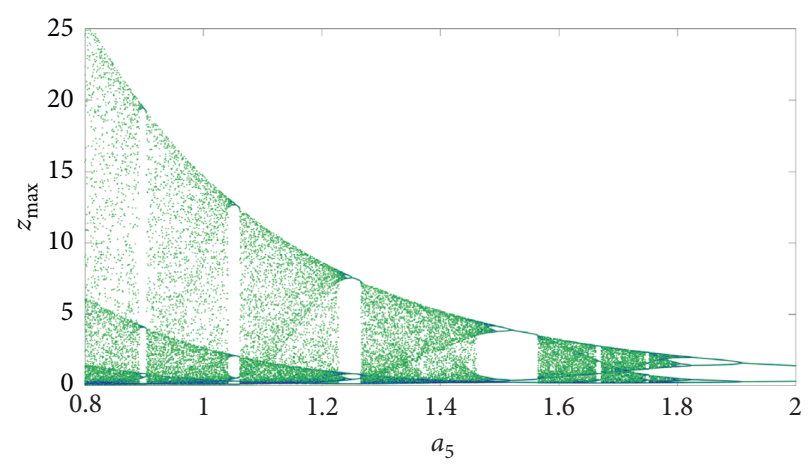

(a)

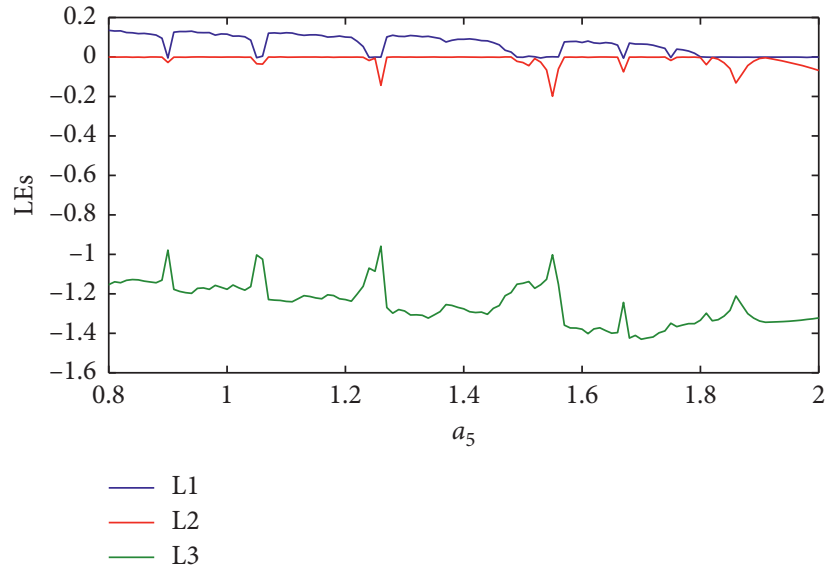

(b)

Figure 4: (a) Bifurcation of the system with $a_{5}$; (b) corresponding LEs.

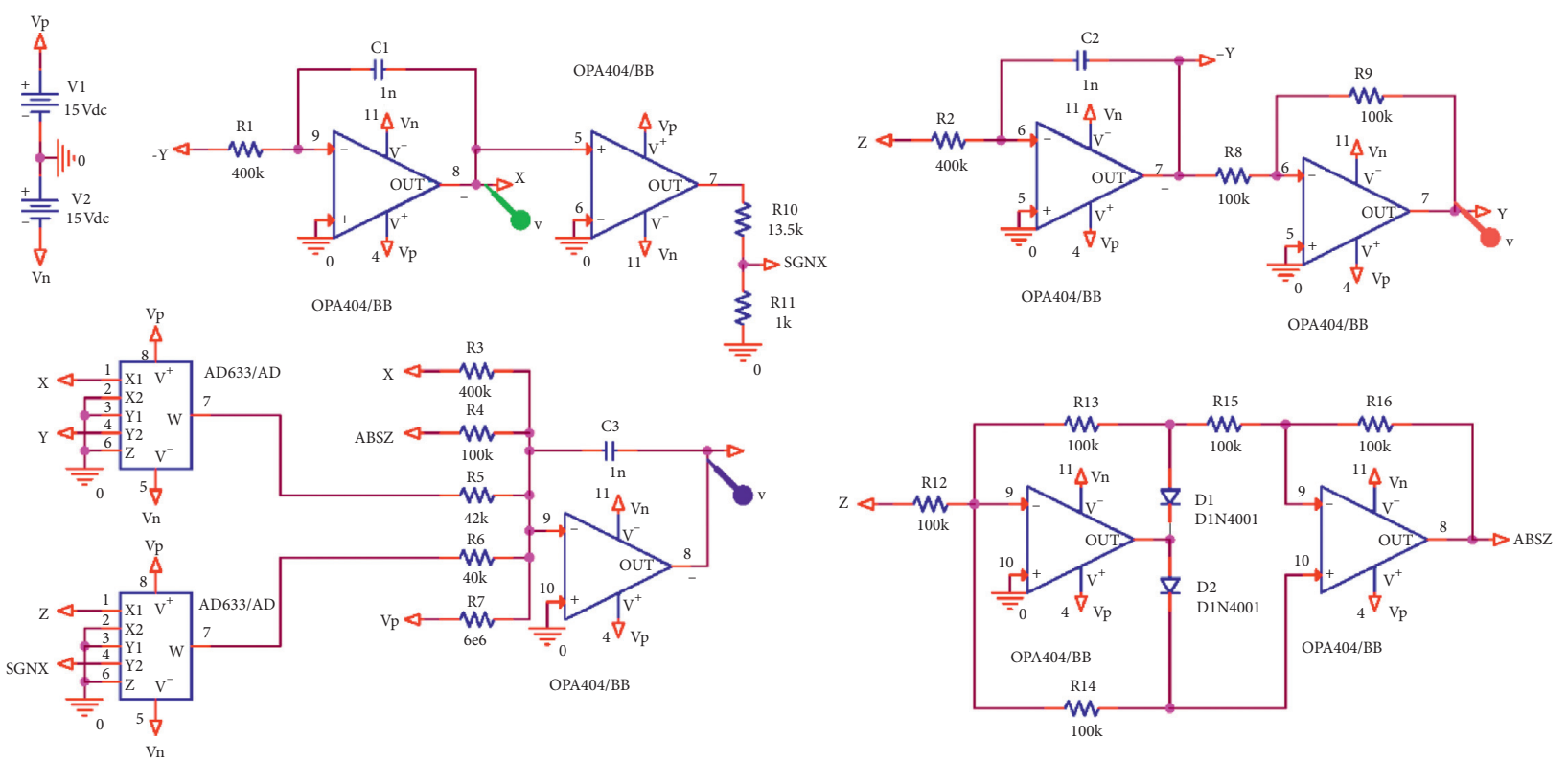

Figure 5: The designed circuit for system (1).
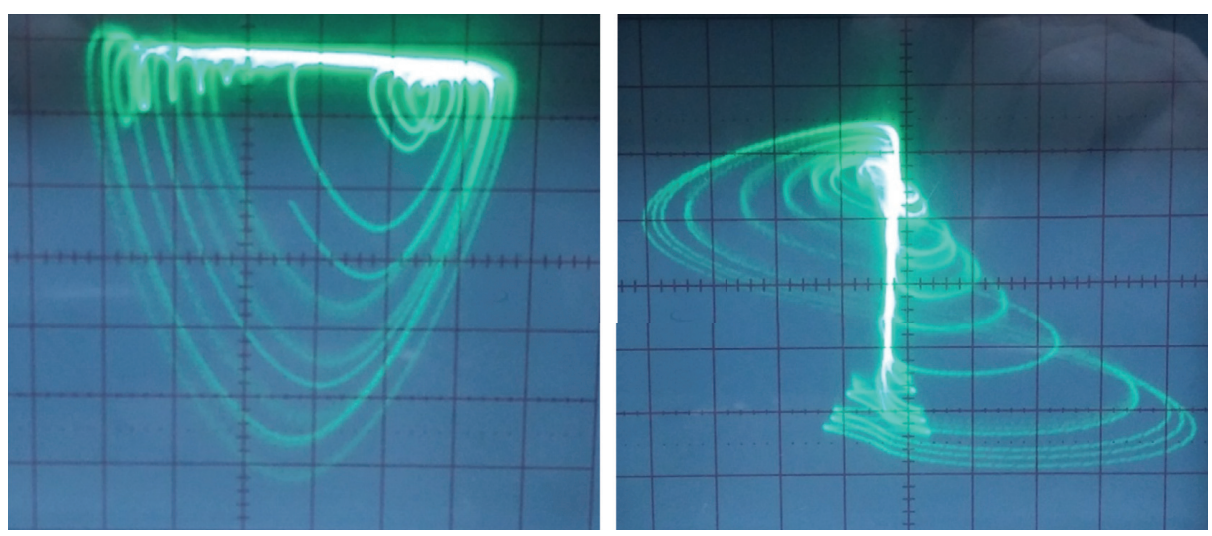

(a)

Figure 6: Continued. 

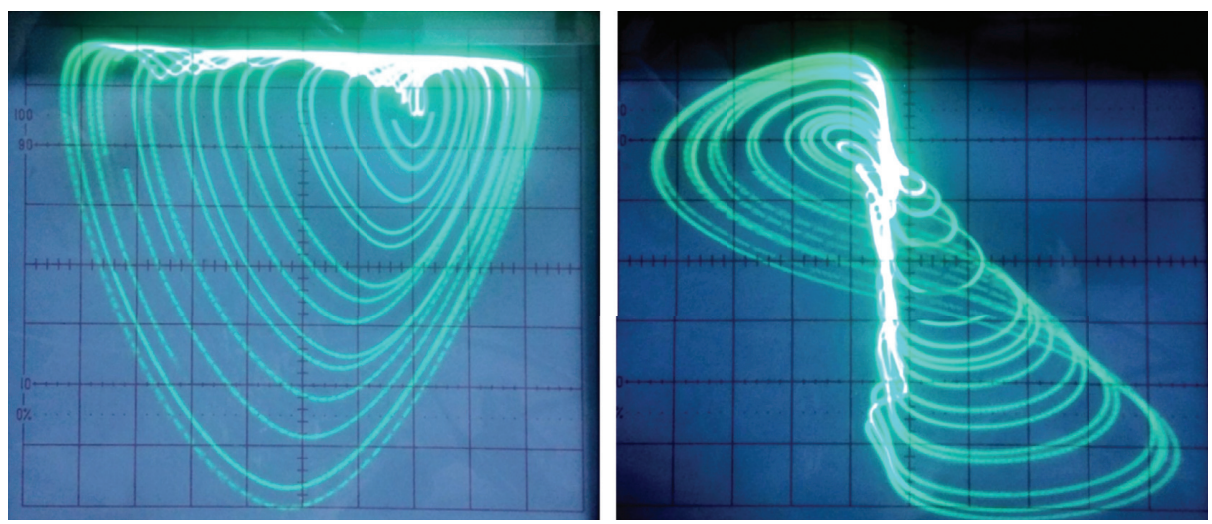

(b)

Figure 6: 2D phase portraits, obtained from the oscilloscope, of the novel system for $a_{1}=1, a_{2}=1, a_{3}=-1, a_{4}=-4$, and $a_{6}=1$ and initial conditions $\left[\begin{array}{lll}-3 & 4 & -4\end{array}\right]$ (a) when $a_{5}=0.95$ and $a_{7}=-1$ and (b) when $a_{5}=1.6$ and $a_{7}=-1$.

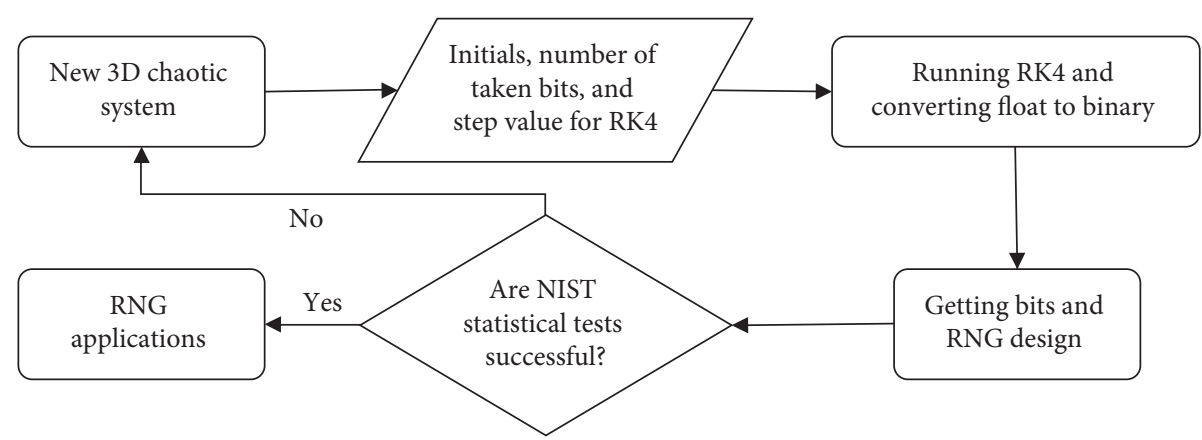

FIGURE 7: RNG design stages with the proposed 3D chaotic system.

TABle 2: 3D chaotic system PRNG NIST-800-22 test results.

\begin{tabular}{|c|c|c|c|c|}
\hline Statistical tests & $p$ value $(X \oplus Y \oplus Z)$ & $p$ value $(Y \oplus Z)$ & $p$ value $(Z)$ & Result \\
\hline Frequency (monobit) test & 0.373465 & 0.017981 & 0.485177 & Successful \\
\hline Block-frequency test & 0.932102 & 0.563486 & 0.720823 & Successful \\
\hline Cumulative-sum test & 0.692331 & 0.029619 & 0.813864 & Successful \\
\hline Runs test & 0.093974 & 0.417706 & 0.661739 & Successful \\
\hline Longest-run test & 0.651016 & 0.544020 & 0.730122 & Successful \\
\hline Binary matrix rank test & 0.489777 & 0.123925 & 0.328310 & Successful \\
\hline Discrete Fourier transform test & 0.393422 & 0.291282 & 0.769023 & Successful \\
\hline Nonoverlapping template test & 0.058054 & 0.045477 & 0.115145 & Successful \\
\hline Overlapping template test & 0.245790 & 0.504454 & 0.546871 & Successful \\
\hline Maurer's universal statistical test & 0.550389 & 0.259675 & 0.138921 & Successful \\
\hline Approximate entropy test & 0.173084 & 0.067221 & 0.503813 & Successful \\
\hline Random excursion test $(x=-4)$ & 0.617626 & 0.055541 & 0.042691 & Successful \\
\hline Random-excursion variant test $(x=-9)$ & 0.842508 & 0.227993 & 0.065864 & Successful \\
\hline Serial test- 1 & 0.651632 & 0.454848 & 0.316808 & Successful \\
\hline Serial test- 2 & 0.675012 & 0.598698 & 0.073441 & Successful \\
\hline Linear complexity test & 0.073771 & 0.830488 & 0.259938 & Successful \\
\hline
\end{tabular}

Otherwise, the procedure is repeated by starting over and setting the initial values, the number of bits taken from the state variables, and the step value for RK4. These operations are carried out until the bit array obtained has passed all of the NIST tests. When the process is complete, an RNG is obtained which successfully passed through the NIST tests, which are the most accepted randomness tests in the literature.
The most important randomness test which is accepted internationally is the NIST-800-22 statistical tests. In this test, there are 15 different subtests. An array consisting of at least 1000000 bits must pass all of these tests successfully. That is, the $p$ values obtained at the end of the tests should be equal or greater than 0.001 . Table 2 shows the results of the NIST tests of the arrays which are generated by taking 8 bits 


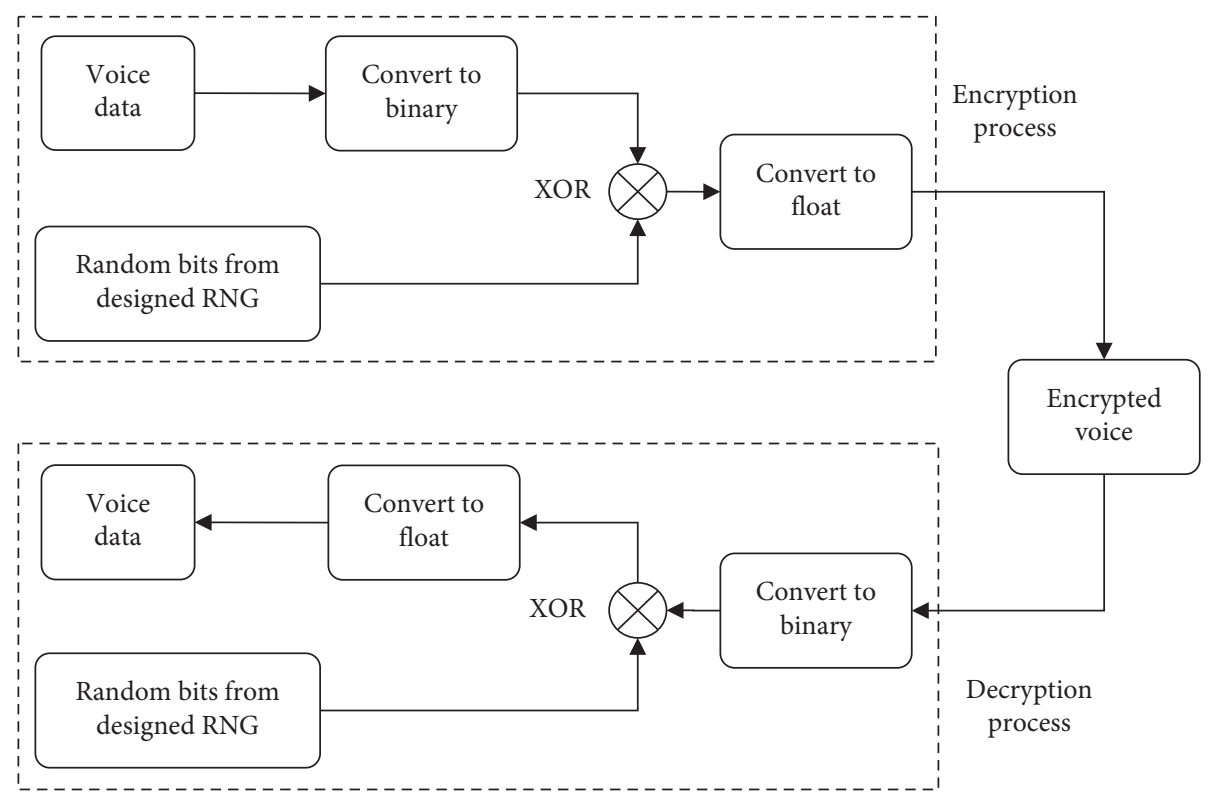

Figure 8: Encryption and decryption process.
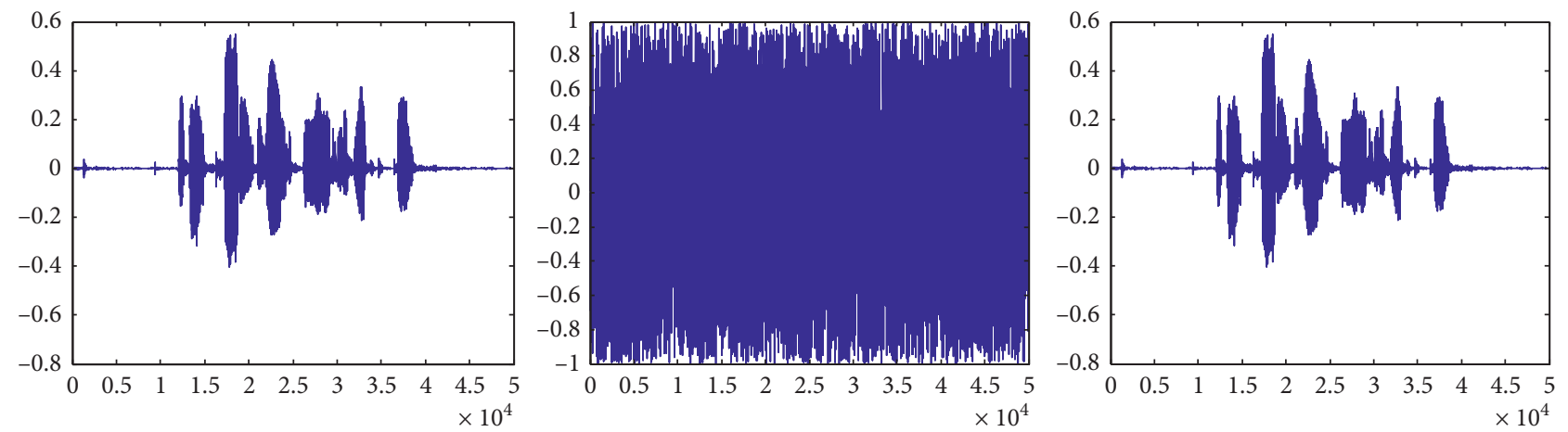

FIGURE 9: Original, encrypted, and decrypted voice waves, respectively.
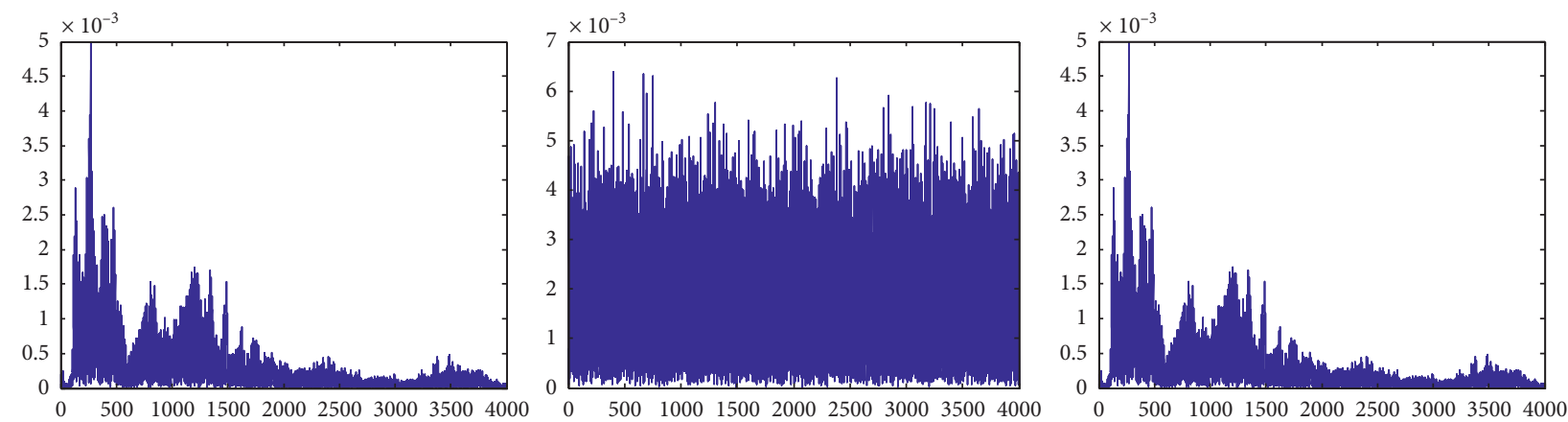

FIGURE 10: Original, encrypted, and decrypted voice spectrums.

from the state variables of the new chaotic system in each iteration. These arrays have successfully passed all the tests. Thus, it has been shown that the proposed system can be used conveniently in RNG-based engineering applications. The arrays are obtained in three different ways, in which the bit arrays obtained from all state variables are subjected to $\mathrm{XOR}$ operation, the $Y$ and $\mathrm{Z}$ state variables are subjected to XOR operation, or using the $Z$ state variable alone.
4.2. Voice Encryption Application. In this part of the study, a different application based on the new chaotic system has been realized. In this application, voice encoding and decoding are performed using the RNG designed in the previous section. The block diagram of the application is shown in Figure 8.

As can be seen from Figure 9, these voice data are recorded with noise, and noise is added in the simulation; 
the voice data are converted into a binary form and then subjected to XOR operation with a random bit array from the RNG. The encrypted bit array is converted back to the float to get the encrypted voice. In the decoding process, the encrypted voice values are converted into binary form and subjected to XOR operation with bit arrays received from the RNG. The bit array obtained after the XOR operation is converted to a float, and the decoding process is completed.

Figure 9 shows the original, encrypted, and decrypted voice plots, respectively. It is seen that there is no similarity between the encrypted voice and the original voice, and that the original voice can be reacquired at the end of the decoding process. The frequency spectrums of the voice signals are shown in Figure 10. When the spectrums are examined, it is seen that the original and decrypted voice spectrums are the same, and the coded spectrum is completely different from the others and has a homogeneous distribution. This indicates that the encryption and decryption processes are quite good.

\section{Conclusion and Discussion}

Signum function and absolute value function can remove the amplitude or polarity information in the feedback variable, which sometimes still preserves the basic property in a dynamical system. As a new case, such a hypogenetic jerk system is obtained, which has stable or unstable equilibrium points under different parameters. Moreover, physical experiments prove the chaotic oscillation. Further discussion focuses on random number generator, and voice encryption shows that the new derived chaotic system still exhibits great noise-like randomness.

\section{Data Availability}

The data that support the findings of this study are available from the corresponding author upon reasonable request.

\section{Conflicts of Interest}

The authors declare that they have no conflicts of interest.

\section{Acknowledgments}

This work was supported financially by the National Nature Science Foundation of China (Grant no. 61871230), the Natural Science Foundation of Jiangsu Province (Grant no. BK20181410), and a project funded by the Priority Academic Program Development of Jiangsu Higher Education Institutions.

\section{References}

[1] S. Lian, "Efficient image or video encryption based on spatiotemporal chaos system," Chaos, Solitons \& Fractals, vol. 40, no. 5, pp. 2509-2519, 2009.

[2] G. Chen, Y. Mao, and C. K. Chui, "A symmetric image encryption scheme based on 3D chaotic cat maps," Chaos, Solitons \& Fractals, vol. 21, no. 3, pp. 749-761, 2004.
[3] Z. H. Guan, F. Huang, and W. Guan, "Chaos-based image encryption algorithm," Physics Letters A, vol. 346, no. 1-3, 2005.

[4] X. Wang, L. Teng, and X. Qin, "A novel colour image encryption algorithm based on chaos," Signal Processing, vol. 92, no. 4, pp. 1101-1108, 2012.

[5] J. Mou, F. Yang, R. Chu et al., "Image compression and encryption algorithm based on hyper-chaotic map," Mobile Networks \& Applications, no. 3, pp. 1-13, 2019.

[6] A. Akgul, S. Kacar, B. Aricioglu et al., "Text encryption by using one-dimensional chaos generators and nonlinear equations," in Proceedings of the International Conference on Electrical \& Electronics Engineering, Mysore, Karnataka, January 2013.

[7] A. P. Johnson, R. S. Chakraborty, and D. Mukhopadyay, “An improved DCM-based tunable true random number generator for xilinx FPGA," IEEE Transactions on Circuits and Systems II: Express Briefs, vol. 64, no. 4, pp. 452-456, 2017.

[8] T. T. Kim Hue and T. M. Hoang, "Complexity and properties of a multidimensional cat-hadamard map for pseudo random number generation," The European Physical Journal Special Topics, vol. 226, no. 10, pp. 2263-2280, 2017.

[9] S. J. Linz and J. C. Sprott, "Elementary chaotic flow," Physics Letters A, vol. 259, no. 3-4, pp. 240-245, 1999.

[10] J. C. Sprott, "A new class of chaotic circuit," Physics Letters A, vol. 266, no. 1, pp. 19-23, 2006.

[11] J. C. Sprott, "A new chaotic jerk circuit," IEEE Transactions on Circuits and Systems II: Express Briefs, vol. 58, no. 4, pp. 240-243, 2011.

[12] J. C. Sprott, "Simplest dissipative chaotic flow," Physics Letters A, vol. 228, no. 4-5, pp. 271-274, 1997.

[13] J. C. Sprott, "Some simple chaotic jerk functions," American Journal of Physics, vol. 65, no. 6, pp. 537-543, 1997.

[14] C. Li, J. C. Sprott, and H. Xing, "Hypogenetic chaotic jerk flow," Physics Letters A, vol. 380, no. 11-12, 2016.

[15] K. Rajagopal, V. T. Pham, F. R. Tahir et al., "A chaotic jerk system with non-hyperbolic equilibrium: dynamics, effect of time delay and circuit realization," Pramana, vol. 90, no. 4, p. 52, 2018.

[16] Q. Lai and S. Chen, "Coexisting attractors generated from a new 4D smooth chaotic system," International Journal of Control, Automation and Systems, vol. 14, no. 4, pp. 11241131, 2016.

[17] R. Guo, "Projective synchronization of a class of chaotic systems by dynamic feedback control method," Nonlinear Dynamics, vol. 90, no. 1, 2017.

[18] Z. Wang and R. Guo, "Hybrid synchronization problem of a class of chaotic systems by an universal control method," Symmetry, vol. 10, no. 11, p. 552, 2018.

[19] X. Yi, R. Guo, and Y. Qi, "Stabilization of chaotic systems with both uncertainty and disturbance by the UDE-based control method," IEEE Access, vol. 8, 2020.

[20] L. Liu, R. Guo, J. Ji, Z. Miao, and J. Zhou, "Practical consensus tracking control of multiple nonholonomic wheeled mobile robots in polar coordinates," International Journal of Robust and Nonlinear Control, pp. 1-17, 2020.

[21] S. Zhou and X. Wang, "Identifying the linear region based on machine learning to calculate the largest lyapunov exponent from chaotic time series," Chaos: An Interdisciplinary Journal of Nonlinear Science, vol. 28, no. 12, Article ID 123118, 2018.

[22] S. Zhou, X. Wang, Z. Wang, and C. Zhang, "A novel method based on the pseudo-orbits to calculate the largest lyapunov exponent from chaotic equations," Chaos, vol. 29, no. 3, Article ID 033125, 2019. 
[23] C. Li, T. Lu, G. Chen, and H. Xing, "Doubling the coexisting attractors," Chaos, vol. 29, no. 5, Article ID 051102, 2019.

[24] C. Li, Y. Xu, G. Chen et al., "Conditional symmetry: bond for attractor growing," Nonlinear Dynamics, vol. 95, no. 2, 2018.

[25] X. Wang and G. Chen, "A chaotic system with only one stable equilibrium," Communications in Nonlinear Science and Numerical Simulation, vol. 17, no. 3, pp. 1264-1272, 2012.

[26] S. Jafari, J. C. Sprott, and M. Molaie, "A simple chaotic flow with a plane of equilibria," International Journal of Bifurcation and Chaos, vol. 26, no. 6, Article ID 1650098, 2016.

[27] S. Jafari, A. Ahmadi, A. J. M. Khalaf et al., "A new hidden chaotic attractor with extreme multi-stability," Aeu International Journal of Electronics \& Communications, vol. 89, pp. 131-135, 2018.

[28] B. Hamdi and S. Hassen, "A new hypersensitive hyperchaotic system with no equilibria," International Journal of Bifurcation and Chaos, vol. 27, no. 5, Article ID 1750064, 2017.

[29] X. Y. Hu, C. X. Liu, L. Liu, Y. P. Yao, and G. C. Zheng, "Multiscroll hidden attractors and multi-wing hidden attractors in a 5-dimensional memristive system," Chinese Physics B, vol. 26, no. 11, pp. 124-130, 2017.

[30] K. E. Chlouverakis and J. C. Sprott, "Chaotic hyperjerk systems," Chaos, Solitons \& Fractals, vol. 28, no. 3, pp. 739-746, 2006. 